\title{
Frei bleiben
}

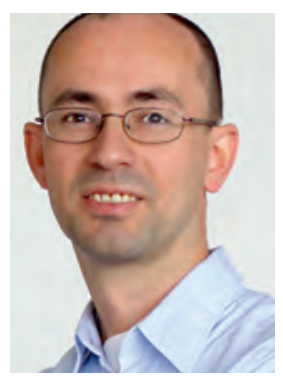

Möchtest du in deiner Arbeit das Maximum dessen einbringen, was die Wissenschaft hergibt? Dann ist Intensivmedizin für dich ideal. Unser aktueller Facharztcheck(S. 22) zeigt: Diese Hightech-Wiese der Medizin trägt ihren Namen zu Recht. Andererseits: Ist denn die Medizin, die in anderen Fächern praktiziert wird, weniger „intensiv“? Jeder Arzt, der Spaß an seiner Arbeit hat, bringt sich intensiv ein - egal ob als Chirurg, als Urologe oder als Hausarzt. Wobei Ärzte allerdings immer aufpassen sollten, es mit ihrem intensiven Einsatz nicht zu übertreiben. Beispiel: Eine US-Studie zeigte kürzlich, dass eine Begrenzung der Arbeitszeiten bei Chirurgen zu mehr Komplikationen bei den Patienten und einer schlechteren Ausbildung führt [1]. Prompt nahmen deutsche Chirurgen diese Studie gleich zum Anlass, zu fragen, wie sinnvoll der Wunsch junger Kollegen nach mehr Freizeit überhaupt ist. So eine Denkweise ist typisch - und gefährlich. Denn: Ja, Ärzte, die früher nach Hause gehen, lernen langsamer. Ja, eventuell leidet darunter sogar die Versorgung. Das ist bitter. Aber dann muss dieser Qualitätsverlust eben durch mehr Investitionen z. B. in Personal ausgeglichen werden - und nicht durch Verzicht auf Familie und Freizeit. Denn unser Leben gehört uns! Sich intensiv der Medizin zu widmen, ist etwas Wunderbares - aber nur dann, wenn es jederzeit eine freie Entscheidung bleibt. 